\title{
RESUMEN
}

La facturación electrónica se ha involucrado con el avance tecnológico y la implementación de sistemas operativos en el ámbito empresarial, el mismo que ayudó a la implementación de controles contables de una entidad debido a que la automatización de programas ha permitido mejorar los medios de comunicación entre varias receptores al mismo tiempo, mediante el estudio realizado en el sector carrocero del Cantón Ambato se pudo identificar que ha sido un medio de control para el Estado en el ámbito tributario concluyendo que gracias al ahorro de tiempo que se ha generado con la implementación de la misma se ha podido mejorar el cumplimiento de las obligaciones tributarias, además se ha realizado un estudio entre las cuentas y rubros que intervienen en la facturación tanto física como electrónica, pudiendo obtener como resultado que la segunda otorga más beneficios que desventajas en el área tributaria.

Palabras clave: facturación electrónica, cumplimiento tributario, sector carrocero, crecimiento tecnológico.

\section{FACTURACIÓN ELECTRÓNICA UN MECANISMO DE CONTROL PARA EL CUMPLIMIENTO TRIBUTARIO, CASO SECTOR CARROCERO.}

\author{
Mauricio Arias Pérez, / Andrea Cáceres Ortiz
}

Fechas de recepción: 15 de Septiembre de 2021

Fecha de aceptación: 07 de Diciembre de 2021

DOl: http://doi.org/10.22370/riace.2021.10.1.2987

1 Facultad de Contabilidad y Auditoría, Universidad Técnica de Ambato, Ambato, Ecuador.

mauriciogarias@uta.edu.ec

2 Ambato, Ecuador, andrea6497_@hotmail.com 


\title{
ELECTRONIC INVOICING A CONTROL MECHANISM FOR TAX COMPLIANCE, CASE BODYBUILDING SECTOR.
}

\begin{abstract}
Electronic invoicing has been involved with technological advancement and the implementation of operating systems in the business environment, which helped the implementation of accounting controls of an entity because the automation of programs has made it possible to improve the means of communication between several recipients at the same time, through the study carried out in the bodybuilding sector of Canton Ambato, it was possible to identify that it has been a means of control for the State in the tax field, concluding that thanks to the time savings generated by the implementation of the same, it has been possible to improve compliance with tax obligations, a study between the accounts and items involved in both physical and electronic invoicing, with the result that the latter provides more benefits than disadvantages in the tax area.
\end{abstract}

Keywords: electronic invoicing, tax compliance, bodybuilding sector, technological growth. 


\section{INTRODUCCIÓN}

La facturación electrónica ha sido considerada como un proyecto de ayuda para el cumplimiento tributario a nivel mundial lo que ha permitido que varios países se acojan a esta nueva modalidad de facturación en las empresas permitiendo a cada uno de ellos por este medio alcanzar una mayor recaudación de impuestos.

La facturación electrónica da su origen con la aparición de tecnologías de información y comunicación. En el año 1945 después de terminar con las alianzas entre Estados Unidos y la antigua Unión Soviética comienza la recordada Guerra Fría terminando hasta el año 1989, en este lapso se decide crear un producto llamado ARPANET conformado por 4 miembros muy importantes como lo son Universidad de California Los Ángeles (UCLA), Universidad de California Santa Bárbara (UCSB), Universidad de Utah y Stanford Research Institute (SRI), con el objetivo de transmitir información. (Jaime, Edisson, \& Adriana, Facturación electrónica versus facturación clásica. Un estudio en el comportamiento financiero mediante estudios de caso, 2016). El objetivo de este estudio es encontrar el impacto que se ha alcanzado con la facturación electrónica después de su implementación en las empresas carroceras motivo de estudio. Se ha podido analizar cuentas que intervienen tanto en la facturación tradicional como en la facturación electrónica pudiendo hacer una comparación sobre la disminución notable que se han dado tanto en gastos, en la captación de información y demás beneficios que se pueden notar como la mejora del cumplimiento tributario, información que ha sido corroborada gracias al levantamiento de información en las entidades mencionadas, y de esta manera compartir en este estudio los resultados alcanzados.

Los tributos ha sido una gestión que ha evolucionado desde mediados del siglo pasado; sin embargo, se espera que por medio de la digitalización acelere el proceso de este. A partir de la consolidación de lo llamado estados nacionales, se consideró al arancel de importación como un tributo necesario. Sin embargo, a finales de la Segunda Guerra Mundial, se considera desarrollar entidades de administración de impuestos en América Latina. (Barreix \& Zambrano, 2018).

Se busca hablar de tributos podemos mencionar que en un sin número de países se han adaptado a esa modalidad para la captación de los mismos y se ha recurrido a documentos que nos permitan verificar la legalidad y cumplimiento de los mismos, por ello es que para el ente regulador de impuestos en el Ecuador el comprobante electrónico pasa a ser un documento que está bajo los requisitos legales y reglamentarios que se necesitan para convertirse en comprobantes de venta, el cual le garantiza la originalidad y procede a ser emitido mediante la modalidad electrónica. (Servicio de Rentas Internas, 2019).

La facturación electrónica se le consideró como una técnica para generar documentos tributarios los mismos que pasarían a ser un remplazo del documento físico tradicional, debido a que la funcionalidad es la misma el recolectar 
información relativa de la transacción, obligaciones de pago y la liquidación de impuestos respectiva. (Hernandez \& Serrano, 2019).

La facturación electrónica se fue extendiendo por toda América Latina y los distintos países se fueron acogiendo de acuerdo con cada uno de los objetivos planteados por las distintas Administraciones Tributarias como, por ejemplo:

Argentina: a principios de los años 90 se daba ya la obligación de registrar las transacciones mediante comprobantes, sin ser en su momento tan rigurosa. Para el año 2005 el régimen decide instrumentar el sistema que emita ya electrónicamente, remplazando el papel. En el 2007 ya se generó el cronograma de obligatoriedad, con el objetivo de que el Fisco conozca inmediatamente los datos de la transacción.

Brasil: en este país se añade en el 2003 un modelo que existe hasta la fecha en donde el contribuyente emite mensualmente el archivo con todas las facturas emitidas en ese periodo.

Chile: El SIl en el 2002 expandió a algunos contribuyentes la idea de realizar un plan piloto con la participación de 8 empresas. Para esto en el año 2003 inicia ya el proceso de forma voluntaria llegando a su fin en el 2014 en donde se rige la Ley 20.727 de obligatoriedad de los contribuyentes con sus respectivas excepciones. (Barreix \& Zambrano, 2018)

Ecuador: En base a la resolución N NACDGERCGC12-00105 creada en el año 2012 procede a constar las normativas para la emisión de comprobantes de venta, documentos de retención y complementarios como documentos electrónicos empezando como un proyecto voluntario por parte de los contribuyentes, pero a partir del año 2013 se crea la resolución respectiva en donde empieza a obligarse a los distintos contribuyentes de acuerdo a sus diferentes actividades económicas a las que se dedican. (Coca \& Flores, 2020)

En Ecuador se tuvo como primordiales objetivos para la Administración Tributaria Ios siguientes:

- Crear un modelo de emisión de documentos para clientes externos de la Administración Tributaria.

- Mejora de operaciones

- Diminución de costos operativos y administrativos en las empresas.

Debido a que el Servicio de Rentas Internas busca fortalecer el comercio electrónico Ecuador, decide impulsar un nuevo modelo de tecnología especialmente para las pequeñas y medianas empresas, para la posterior mejora en la eficiencia y competitividad, (Cáceres, 2020). Se creó este proyecto mediante tres etapas en donde los contribuyentes debían certificarse para emitir las mismas que menciona Barreix y Zambrano (2018) como: 
- Etapa de desarrollo: los contribuyentes crean su propio esquema de facturación acoplando a su sistema contable. El SRI creó un sistema genérico para los contribuyentes que no podían desarrollar el sistema propio.

- Etapa de prueba: después de contar con una infraestructura tecnológica se certifica a los contribuyentes para el periodo de prueba, en donde se emitían comprobantes electrónicos sin validez legal, debido a que el fin era solo conocer la velocidad de interconexión la consistencia de información y como responde el sistema tecnológico.

- Etapa de producción: después de haber pasado por estas dos etapas principales, el SRI procede a emitir la certificación para iniciar la emisión formal de los comprobantes en las distintas transacciones ya sea de consumo intermedio o final de la empresa.

\section{Facturación electrónica}

A pesar de que los países han implementado en distintos años la facturación electrónica este ha sido un proceso paulatino para todos. Por ello en las primeras etapas de piloteo e implementación el porcentaje de los contribuyentes utilizando la Facturación Electrónica era bajo para cuando se dio los plazos de obligatoriedad.

Después de que las distintas administraciones tributarias ponen en marcha el proyecto en sus contribuyentes se tiene una aceleración considerable en la implementación de está llegando hasta un 100\% de contribuyentes que emiten electrónicamente por lo cual se muestra en el siguiente gráfico.

\section{Ilustración I Contribuyentes del IVA que emiten Factura Electrónica}

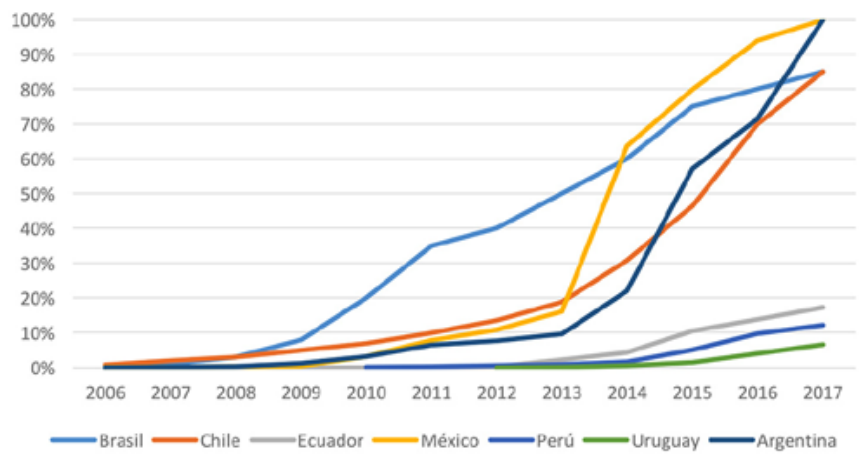

Fuente: Cáceres (2020)

Se destaca que en Ecuador para el año 2017 ya se tenía cerca de un 20\% de las empresas emitiendo comprobantes de manera electrónica mientras que países como México y Argentina habían alcanzado un 100\% en su facturación electrónica lo que deja ver que América Latina ha recibido de forma positiva este proyecto. 


\section{Documentos electrónicos}

Un documento electrónico se convierte en un comprobante que cumple con todas las necesidades legales y reglamentarias tanto que así se encuentren como un documento legal de origen e integridad de contenido. Para ser un documento electrónico debe cumplir con los siguientes requisitos que establece el Servicio de Rentas Internas:

- Firma electrónica

- Software que genere comprobantes electrónicos (puede ser propio 0 se puede utilizar la herramienta de comprobantes electrónicos de uso gratuito)

- Conexión a Internet

- $\quad$ Clave de acceso al SRI en Línea

- Convenio de débito registrado

Entre los documentos que se pueden emitir de forma electrónica se puede obtener Ios siguientes:

\section{Tabla I Documentos Electrónicos}

\section{DOCUMENTO}

Facturas

Destinadas a sociedades 0 personas naturales que tengan derecho a crédito tributario y en operaciones de exportación

\begin{tabular}{ll}
\hline $\begin{array}{l}\text { Liquidaciones de } \\
\text { compras de bienes y } \\
\text { prestación de servicios }\end{array}$ & $\begin{array}{l}\text { Las emiten en servicios o adquisiciones de acuerdo con las } \\
\text { condiciones previstas en el Reglamento. }\end{array}$ \\
\hline Notas de crédito & $\begin{array}{l}\text { Se emiten para anular operaciones, aceptar devoluciones y } \\
\text { conceder descuentos o bonificaciones. }\end{array}$ \\
\hline Notas de débito & $\begin{array}{l}\text { Se emiten para cobrar intereses de mora y para recuperar } \\
\text { costos y gastos }\end{array}$ \\
\hline $\begin{array}{l}\text { Comprobantes de } \\
\text { retención }\end{array}$ & Comprobantes que acreditan la retención del impuesto \\
\hline Guías de remisión & Sustenta el traslado de mercaderías dentro del territorio nacional \\
\hline
\end{tabular}

Fuente: Cáceres (2020)

\section{Firma electrónica}

Las facturas electrónicas contienen una firma digital la misma que es autenticada por el emisor por lo cual no se permite el rechazo del documento, es necesario que el documente cuente con los parámetros suficientes para que este sea válido y garantizado, para todo esto la firma debe ser autorizada por una plataforma digital y por la propia Administración Tributaria. (Barreix y otros, 2018) 
Existen tres tipos importantes de firmas electrónicas entre ellas se tienen a las siguientes:

\section{Tabla II Tipos de firmas}

\begin{tabular}{|c|c|c|}
\hline Firma Básica & Firma Fichada & Firma Completa \\
\hline $\begin{array}{l}\text { Recoge elementos } \\
\text { básicos de la Facturación } \\
\text { electrónica, donde incorpora } \\
\text { el resumen del documento, } \\
\text { el certificado del firmante } \\
\text { con su clave }\end{array}$ & $\begin{array}{l}\text { Añade información sobre } \\
\text { vigencia del certificado en } \\
\text { el momento de la firma y } \\
\text { verificación }\end{array}$ & $\begin{array}{l}\text { Incluye todos los elementos } \\
\text { para verificar la vigencia del } \\
\text { certificado en el momento } \\
\text { de la firma }\end{array}$ \\
\hline
\end{tabular}

Fuente: Díaz, Coba \& Bombón (2016)

\section{Ventajas de la facturación electrónica}

De acuerdo con Chávez (2020) se menciona las siguientes ventajas:

- Ahorro de tiempo de envío del documento, fortaleciendo los lazos de comunicación entre emisor y receptor.

- Ahorro de gasto de insumos para emisión de un documento tradicional, entre ellos compra de papel, tinta, entre otros.

- Liberación de espacios físicos que se utilizaban para el archivo de documentos.

- Menos error de contenido y menor riesgo de alteración de un documento.

- Aceleración del proceso administrativo por el acceso a un documento por parte de todos los funcionarios.

- Ayuda al cuidado del medio ambiente, por la reducción en la utilización del papel.

\section{METODOLOGÍA}

Para la presente investigación se recolecto información veraz de algunas fuentes permitiendo realizar el análisis correspondiente y poder determinar los resultados pertinentes, realizando encuestas elaboradas previamente, las mismas que serán dirigidas a los representantes del área contable o quienes estén directamente relacionados con la facturación electrónica en las empresas seleccionadas del sector carrocero del Cantón Ambato quienes son con los que se van a analizar en el presente caso. Por medio de esto permitirá conocer cuáles han sido los mecanismos de control que se han visto más favorecidos en la empresa y también como ha mejorado el cumplimiento tributario con la respectiva Administración Tributaria. 
Se dispone de información a través de los Estados Financieros de las empresas a estudiar por su publicación en la página oficial de la Superintendencia de Compañías, en donde se procederá a realizar el respectivo análisis de las cuentas que intervienen en el proceso de facturación y poder ver cuál ha sido el impacto de ahorro con la facturación electrónica frente a la facturación tradicional, teniendo en cuenta que para este estudio se ha considerado las empresas con denominación sociedades y que se encuentran dentro del sector carrocero, para lo cual se procede a estudiar el formulario, en donde se declara obligatoriamente cada año su respectivo impuesto a la renta.

\section{RESULTADOS}

Como se ha mencionado anteriormente se puede constatar que se ha realizado un análisis de las diferentes cuentas que intervienen en la facturación electrónica y las mismas que permitirá conocer si el ahorro en las empresas se ha dado desde una primera instancia. Para ello se ha decidido estudiar las siguientes cuentas:

Tabla III Variables consideradas

\begin{tabular}{|c|c|}
\hline CUENTAS & CASILLEROS \\
\hline \multicolumn{2}{|c|}{ Formulario 101} \\
\hline $\begin{array}{l}\text { Suministros, herramientas, } \\
\text { materiales }\end{array}$ & 7191 \\
\hline Mantenimiento & 7197 \\
\hline \multicolumn{2}{|c|}{ Valores Encuestados } \\
\hline Software & Amortización \\
\hline Depreciación Equipo Computo & Sueldos y Salarios. \\
\hline
\end{tabular}

Fuente: Autores

Para el análisis se procedió a utilizar los valores declarados en el casillero correspondiente, en concordancia con el porcentaje perteneciente de cada empresa de acuerdo con la ponderación en la opinión de cada uno de los representantes que conocen los datos de cada organización. Posterior a ello se ha presentado la cantidad de documentos emitidos en cada periodo fiscal. 
Tabla IV Ahorro de Facturación

\begin{tabular}{|c|c|c|c|c|c|}
\hline & 101 & $\begin{array}{l}\text { CEPEDA } \\
\text { CIA. LTDA. }\end{array}$ & $\begin{array}{c}\text { PATRICIO } \\
\text { CEPEDA } \\
\text { CIA. LTDA. }\end{array}$ & $\begin{array}{c}\text { PICO } \\
\text { SÁNCHEZ } \\
\text { CIA. LTDA. }\end{array}$ & $\begin{array}{l}\text { DAVMOTORS } \\
\text { CIA. LTDA. }\end{array}$ \\
\hline \multicolumn{6}{|c|}{ COSTO FACTURACIÓN TRADICIONAL } \\
\hline SOFTWARE & & $\$-$ & $\$-$ & $\$-$ & $\$-$ \\
\hline AMORTIZACIÓN & & $\$-$ & $\$-$ & $\$-$ & $\$-$ \\
\hline $\begin{array}{l}\text { SUMINISTROS, } \\
\text { HERRAMIENTAS, } \\
\text { MATERIALES }\end{array}$ & 7191 & $\$ 12.764,58$ & $\$ 15.779,78$ & $\$ 2.135,01$ & $\$ 966,88$ \\
\hline $\begin{array}{l}\text { EQUIPO DE } \\
\text { COMPUTO }\end{array}$ & & $\$ 3.200,00$ & $\$ 2.000,00$ & $\$ 3.600,00$ & $\$ 1.700,00$ \\
\hline DEPRECIACIÓN & & $\$ 1.066,67$ & $\$ 666,67$ & $\$ 1.200,00$ & $\$ 566,67$ \\
\hline MANTENIMIENTO & 7197 & $\$ 1.112,35$ & $\$ 9.788,15$ & $\$ 838,28$ & $\$ 7.397,75$ \\
\hline $\begin{array}{l}\text { SUELDOS Y } \\
\text { SALARIOS }\end{array}$ & & $\$ 2.400,00$ & $\$ 1.600,00$ & $\$ 3.200,00$ & $\$ 1.600,00$ \\
\hline TOTAL & & $\$ 19.476,93$ & $\$ 29.167,93$ & $\$ 9.773,29$ & $\$ 11.664,63$ \\
\hline $\begin{array}{l}\text { COSTO UNITARIO } \\
\text { POR DOCUMENTO }\end{array}$ & & $\$ 2,43$ & $\$ 4,86$ & $\$ 4,89$ & $\$ 3,05$ \\
\hline \multicolumn{6}{|c|}{ COSTO FACTURACIÓN ELECTRÓNICA } \\
\hline SOFTWARE & & $\$-$ & $\$-$ & $\$ 800,00$ & $\$ 600,00$ \\
\hline AMORTIZACIÓN & & $\$-$ & $\$-$ & $\$ 400,00$ & $\$ 200,00$ \\
\hline $\begin{array}{l}\text { SUMINISTROS, } \\
\text { HERRAMIENTAS, } \\
\text { MATERIALES }\end{array}$ & 7191 & $\$ 4.553,62$ & $\$ 24.352,73$ & $\$ 3.346,28$ & $\$ 2.855,78$ \\
\hline $\begin{array}{l}\text { EQUIPO DE } \\
\text { COMPUTO }\end{array}$ & & $\$ 3.200,00$ & $\$ 2.000,00$ & $\$ 3.600,00$ & $\$ 1.700,00$ \\
\hline DEPRECIACIÓN & & $\$ 1.066,67$ & $\$ 666,67$ & $\$ 1.200,00$ & $\$ 566,67$ \\
\hline MANTENIMIENTO & 7197 & $\$-$ & $\$-$ & $\$ 356,18$ & $\$ 848,39$ \\
\hline $\begin{array}{l}\text { SUELDOS Y } \\
\text { SALARIOS }\end{array}$ & & $\$ 2.400,00$ & $\$ 1.600,00$ & $\$ 3.200,00$ & $\$ 1.600,00$ \\
\hline TOTAL & & $\$ 8.020,28$ & $\$ 26.619,39$ & $\$ 9.302,46$ & $\$ 6.670,84$ \\
\hline $\begin{array}{l}\text { COSTO UNITARIO } \\
\text { POR DOCUMENTO }\end{array}$ & & $\$ 1,00$ & $\$ 4,44$ & $\$ 4,65$ & $\$ 1,75$ \\
\hline AHORRO & & $\$ 1,43$ & $\$ 0,42$ & $\$ 0,24$ & $\$ 1,30$ \\
\hline
\end{tabular}

Fuente: Cáceres (2020)

Tabla V Oportunidad de Ahorro

\begin{tabular}{|l|r|r|r|}
\hline ENTIDAD & FACT. TRADICIONAL & FACT. ELECTRÓNICA & AHORRO \\
\hline CEPEDA CIA. LTDA. & $\$ 2,43$ & $\$ 1,00$ & $\$ 1,43$ \\
\hline PATRICIO CEPEDA CIA. LTDA. & $\$ 4,86$ & $\$ 4,44$ & $\$ 0,42$ \\
\hline PICOSA CIA LTDA. & $\$ 4,89$ & $\$ 4,65$ & $\$ 0,24$ \\
\hline DAVMOTORS S. A & $\$ 3,05$ & $\$ 1,75$ & $\$ 1,30$ \\
\hline
\end{tabular}

Fuente: Cáceres (2020) 
Como se puede ver la oportunidad de ahorro ha sido de forma positiva para todas las empresas en donde queda claro que con la implementación de la facturación electrónica si se ha alcanzado un ahorro pese a que algunas de ellas tuvieron que realizar una inversión inicial por el software que no se adaptaba a los requerimientos necesarios para la emisión de los comprobantes de forma digital, sin embargo se supo reemplazar ese valor frente al ahorro de otro como lo es la cantidad de papel que se necesitaba de forma anual para la entrega de documentos a sus clientes, entre otros.

En el análisis de los valores tanto de la facturación tradicional con la facturación electrónica es posible verificar que el ahorro se genera en la cuenta de suministros de oficina debido a que la empresa optó por reducir los suministros de forma anual gracias a la implementación de su facturación electrónica.

\section{DISCUSIÓN DE RESULTADOS}

Frente al análisis realizado se puede observar el beneficio que se genera en las empresas al momento de realizar las adquisiciones de suministros, lo cual deja ver como un modelo de aceptación para estas empresas frente a las demás gracias a la facturación electrónica, lo cual permite saber que este proyecto sin duda ha traído cambios económicos con la incorporación de esta.

Para ello también se realizó un encuesta en donde se requiere conocer la opinión de los funcionarios específicamente de aquellos que están involucrados en los cambios generados debido a la facturación electrónica, en donde se ha podido llegar a resumir que gracias a la misma la búsqueda de un documento es mucho más fácil, el ahorro que se obtiene a la hora de almacenar el mismo y el tiempo al momento de preparar la información para el cumplimiento respectivo con las obligaciones tributarias, para lo cual se ha disminuido de forma notable errores de contenidos en el documento, declaraciones sustitutivas, anulación de documentos y sanciones económicas por parte de la Administración Tributaria.

\section{CONCLUSIONES}

Este estudio presentado ha sido favorable para todas aquellas empresas que decidieron implementar la facturación electrónica en relación a los datos analizados con valores oficiales de las empresas carroceras del Cantón Ambato que obtuvieron con la implementación del mismo logrando como objetivo primordial de las entidades estudiadas el ahorro económico que se presenta con la aplicación del mismo, sabiendo que no cambiaría ni afectaría de ningún modo el proceso interno de cada institución, por ello se reconoce como un proyecto eficaz no solo para los empresarios sino en general para el país dotando de mecanismos idóneos 
por parte de la Administración Tributaria para el contribuyente de manera que les permita cumplir y mejorar la recaudación fiscal gracias a que se ha podido mejorar el control en las empresas frente al cumplimiento de las obligaciones y más que todo se ha podido evitar que se genere las evasiones de impuestos por parte de ciertos contribuyentes, por eso no hay duda que este mecanismo de facturación se debe incluir en todas las empresas lo más pronto posible y así incrementar el porcentaje de contribuyentes que se unen a esta nueva manera de facturar en nuestro país siendo un estado innovador y tecnológico.

\section{BIBLIOGRAFÍA}

BARREIX, A. D., ZAMBRANO, R., COSTA, M. P., DA SILVA BAHIA, Á. A., DE JESUS, E. A., DE FREITAS, V. P., \& DRAGO, C. (2018). Factura electronica en america latina (Vol. 595). Inter-American Development Bank.

BARREIX, A., \& ZAMBRANO, R. (2018). La Factura Electrónica en América Latina.

CÁCERES, A. (2020). Facturación Electrónica un mecanismo de control para el cumplimiento tributario en el sector carrocero del Canton Ambato. Universidad Técnica de Ambato, Ambato.

CHAVARRÍA, E. J., MOROCHO, L. J., \& AVELLAN, W. W. (2017). Ventajas y desventajas de la implementación de la Facturación electrónica en el Ecuador. RECIAMUC, 1(4), 473-499.

COCA, L., \& FLORES, S. (2020). Facturación electrónica versus facturación clásica. Un estudio en el comportamiento financiero mediante estudios de casos. Ciencia Unemi, 9(18), 63-72.

HERNANDEZ, B. O., \& SERRANO, C. C. (2019). ¿Qué induce a las empresas a adoptar facturación electrónica? Efecto de las percepciones y del entorno competitivo. Universia Business Review, 26.

JAIME, D.-C., EDISSON, C.-M., \& ADRIANA, B.-M. ( 2016). Facturación electrónica versus facturación clásica. Un estudio en el comportamiento financiero mediante estudios de caso. Revista Ciencia UNEMI,Vol. 9 - No 18, Junio, pp. 63 - 72.

JAIME, D.-C., EDISSON, C.-M., \& ADRIANA, B.-M. (Junio de 2016). Facturación electrónica versus facturación clásica. Un estudio en el comportamiento financiero mediante estudios de casos. Revista Ciencia UNEMI, Vol. 9 (No 18), pp. 63 - 72.

MILLET, D., \& NAVARRO, A. (Marzo de 2008). Facturacion electronica: la busqueda de la eficiencia y productividad. Partida Doble, 88-95. Obtenido 
de Partida doble.

NELLY CRISTINA, C. R. (2020). "PLANEAMIENTO TRIBUTARIO EN LAS EMPRESAS DEL SECTOR COMERCIO EN EL PERIODO 2013 - 2019". "PLANEAMIENTO TRIBUTARIO EN LAS EMPRESAS DEL SECTOR COMERCIO EN EL PERIODO 2013 - 2019". UNIVERSIDAD PRIVADA DEL NORTE, Lima.

SÁNCHEZ SERENA, I. (2012). La factura electrónica en España. Revista de Internet, Derecho y Política, 05-12.

SERVICIO DE RENTAS INTERNAS, S. (2019). SRI. Obtenido de SRI. 